\title{
SEMIBLIND CHANNEL ESTIMATION FOR SPACE-TIME CODED WCDMA
}

\author{
Y. Sung and L. Tong ${ }^{\dagger}$ \\ School of Electrical and Computer Engineering \\ Cornell University \\ Ithaca, NY 14850, USA \\ e-mail: \{ys87,ltong $\} @$ ece.cornell.edu
}

\author{
Ananthram Swami \\ Army Research Laboratory \\ 2800 Powder Mill Rd. \\ Adelphi, MD 20783, USA \\ e-mail: a.swami@ieee.org
}

\begin{abstract}
A new semi-blind channel estimation technique is proposed for space-time coded wideband CDMA systems using aperiodic and possibly multirate spreading codes. Exploiting the subspace structure of the WCDMA signaling and the orthogonality of the spacetime code, the proposed algorithm provides the least squares channel estimate in closed form. Using a decorrelating matched filter, the proposed method projects the received signal into subspaces from which channel parameters and data symbols can be estimated jointly. A new blind identifiability condition is established. The mean square error of the estimated channel is compared with the Cramér-Rao bound, and bit error rate (BER) is assessed through Monte Carlo simulation.
\end{abstract}

\section{INTRODUCTION}

Next generation wireless systems are designed for multimedia communication which requires transmissions of high quality images and video. This is especially the case for the downlink where mobile users demand a high volume of data from network servers. To provide reliable communications over time-varying fading channel, diversity techniques in space and time are expected to play a crucial role [1] [4]. A variety of space-time processing or coding schemes have been proposed with multiple transmit antennas and a single or multiple receive antennas (e.g., [2] [3] [5] [6] ). Indeed, the third generation wireless standard supports base station transmit diversity using the WCDMA physical layer.

Many space-time techniques including BLAST and space-time block codes are designed for coherent detection where channel estimation is necessary. There is a substantial literature addressing the channel estimation issue for (space-time coded) multiple-input multiple-output (MIMO) systems, ranging from standard training based techniques that rely on pilot symbols in the data stream to blind and semiblind estimation where observations corresponding to data and pilots (if they exist) are used jointly. Other authors have considered noncoherent detection schemes based on differential encoding which don't require channel state information [7] [9] [8]. Although these methods avoid the need for channel estimation, they often suffer from problems such as error propagation.

In this paper, we focus on space-time coded coherent long code WCDMA systems. The increase in the number of channel parameters, due to the use of multiple antennas, makes the conventional training based scheme less reliable and prone to multiaccess

\footnotetext{
†This work is supported in part by the ARL CTA in Communications and Networks
}

interference. For a reasonable performance, the conventional training based method requires more power and larger number of pilot symbols which reduces system efficiency. Fortunately, WCDMA provides code diversities and signal structure that could be exploited in an estimation scheme. We propose a semiblind channel estimation technique that requires no more than two pilot symbols per user per slot. Using a RAKE structure, our algorithm consists of a standard receiver front-end that suppresses multiaccess interference, and performs single user detection. The proposed algorithm exploits the subspace structure of the long code WCDMA transmission and the orthogonality of the Alamouti code. As a subspace technique, the proposed algorithm can obtain channel estimates quickly using only few symbols, which allows us to deal with fast fading channels.

The paper is organized as follows. The data model of a spacetime coded long code CDMA system is described in Section 2. Section 3 presents a new blind channel estimation method based on a decorrelating matched filter, as well as an identifiability condition and several extensions of the proposed method. In Section 4 , the performance of the proposed method is evaluated through comparison with the Cramér-Rao Bound (CRB) and the bit error rate(BER) is assessed via Monte Carlo simulations.

\section{DATA MODEL}

We consider a space-time coded $K$ user WCDMA system with two transmit antennas and a single receive antenna. We assume that the user signals are synchronized and spread by aperiodic spreading codes of spreading gain $G$, and transmissions are slotted with $M$ symbols per slot.

Specifically, user $i$ 's data stream for each transmit antenna is space-time encoded as [5]

$$
\begin{aligned}
& s_{i, 2 n}^{0}=-s_{i, 2 n-1}^{1 *}, \\
& s_{i, 2 n}^{1}=s_{i, 2 n-1}^{0 *}, \quad n=1,2, \ldots, M / 2
\end{aligned}
$$

where $s_{i, m}^{j} \triangleq s_{i}^{j}\left(m T_{s}\right), \quad j=0,1$, is the $m$ th data symbol of user $i$ for transmit antenna $j, T_{s}$ is the symbol interval, and ( ) ${ }^{*}$ is complex conjugate. Each coded stream is spread by an aperiodic spreading code $c_{i}(t)$ and transmitted from the corresponding antenna. Note that the same code is used by both antennas in figure $1^{1}$.

\footnotetext{
${ }^{1}$ The separation of two antenna signals is possible due to the different sequences for antennas. If different codes are used, the receiver could use both spatial and code information [3].
} 


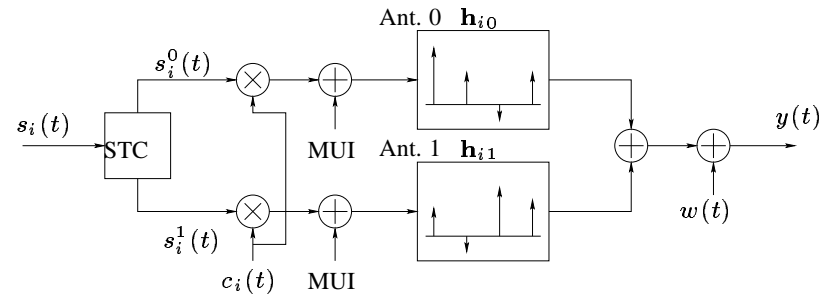

Fig. 1. Downlink Model with Space-time Coding (STC: SpaceTime Block Encoder, MUI:Orthogonal Other User Signal ).

We assume that the channel for each transmitter and receiver pair is a finite length multipath channel with complex path gains separated by multiples of the chip interval and the channel doesn't change over a slot period. The continuous-time impulse response of channel for the $j$ th transmit-receive pair for user $i$ is given by

$$
h_{i j}(t)=\sum_{l=1}^{L_{i j}} h_{i j}^{(l)} \delta\left(t-l T_{c}-d_{i j} T_{c}\right),
$$

where $\left\{h_{i j}^{(l)}\right\}$ is the complex gain for path $l, T_{c}=T_{s} / G$ is the chip duration. We assume that the multipath delays, the $d_{i j}$ 's, are known. When the channel is sparse, it is more efficient to model the channel as separate clusters of fingers. In that case, we assume that the approximate locations of these clusters are known. Since any path gain can be zero, one can over-parameterize the channel to accommodate channel uncertainties. Thus, $L_{i j}$ is often a design parameter. We can set $L_{i j}=L_{i}, \quad j=0,1$ by taking the maximum of $\left\{L_{i j}\right\}$.

At the receiver, the signal $y(t)$ is passed through a chip-matched filter and sampled synchronously at the chip rate. The received noiseless signal vector $\mathbf{y}_{i m}$ that corresponds to the $m$ th symbol interval of user $i$ is given by

$$
\begin{aligned}
\mathbf{y}_{i m} & =\mathbf{T}_{i m}\left[\mathbf{h}_{i 0} s_{i m}^{0}+\mathbf{h}_{i 1} s_{i m}^{1}\right], \\
\mathbf{h}_{i j} & \triangleq\left[h_{i j}^{(1)}, h_{i j}^{(2)}, \cdots, h_{i j}^{(L)}\right]^{T}, j=0,1,
\end{aligned}
$$

where $\mathbf{T}_{i m}$ is the Toeplitz matrix whose first column is made of $(m-1) G+d_{j}$ zeros followed by the code vector $\mathbf{c}_{i m}$-the $m$ th segment of $G$ chips of the spreading code of user $i$-and additional zeros that make the size of $\mathbf{y}_{i m}$ the total number of chips in the entire $M$-symbol slot. The received noiseless signal for user $i$ is given by

$$
\begin{aligned}
\mathbf{y}_{i}= & \sum_{m=1}^{M} \mathbf{T}_{i m}\left[\mathbf{h}_{i 0} s_{i m}^{0}+\mathbf{h}_{i 1} s_{i m}^{1}\right]=\mathbf{T}_{i}\left(\mathbf{I}_{M} \otimes\left[\mathbf{h}_{i 0} \quad \mathbf{h}_{i 1}\right]\right) \mathbf{s}_{i} \\
& \mathbf{s}_{i} \triangleq\left[s_{i 1}^{0}, s_{i 1}^{1}, s_{i 2}^{0}, s_{i 2}^{1}, \cdots, s_{i M}^{0}, s_{i M}^{1}\right]^{T} \\
& \mathbf{T}_{i} \triangleq\left[\mathbf{T}_{i 1}, \mathbf{T}_{i 2}, \cdots, \mathbf{T}_{i M}\right]
\end{aligned}
$$

where $\otimes$ is the Kronecker product and $\mathbf{T}_{i}$ is user $i$ 's code matrix which has a special block shifting structure. Including $K^{\prime}(\leq K)$ dominant users, we have the complete matrix model

$$
\begin{aligned}
\mathbf{y} & =\left[\mathbf{T}_{1} \cdots \mathbf{T}_{K^{\prime}}\right] \operatorname{diag}\left(\mathbf{I}_{M} \otimes \mathbf{H}_{1}, \cdots, \mathbf{I}_{M} \otimes \mathbf{H}_{K^{\prime}}\right) \mathbf{s}+\mathbf{w} \\
& =\mathbf{T} \mathcal{D}(\mathbf{H}) \mathbf{s}+\mathbf{w}
\end{aligned}
$$

where the overall code matrix $\mathbf{T}$ and symbol vector $\mathbf{s}$ contain code matrices and symbols of $K^{\prime}$ dominant users, respectively. Matrix
$\mathcal{D}(\mathbf{H})$ is block diagonal with $\mathbf{I}_{M} \otimes \mathbf{H}_{i}$ as the block element and $\mathbf{H}_{i} \triangleq\left[\mathbf{h}_{i 0} \mathbf{h}_{i 1}\right]$. The additive noise is denoted by $\mathbf{w}$ which may include the users not modeled in the signal part.

We will make the following assumptions.

A1: The code matrix $\mathbf{T}$ is known.

A1': The code matrix $\mathbf{T}$ has full column rank.

A2: The noise vector is complex Gaussian $\mathbf{w} \sim \mathcal{N}\left(\mathbf{0}, \sigma^{2} \mathbf{I}\right)$ with possibly unknown variance $\sigma^{2}$.

Assumption (A1) implies that the receiver knows the codes for $K^{\prime}$ dominant users of interest (as well as the delay $d_{i j}$ and the maximum channel order $L_{i}$ ). For the downlink, the relative delays $d_{i j}$ and the number of multipaths $L_{i}$ are the same for all the user signals. Assumption (A1') is sufficient but not necessary for the channel to be identifiable. This condition is easily satisfied with a relative large spreading gain and proper choice of $K^{\prime}$. When (A1') fails, the channel may still be identifiable as shown in Section 3. In assumption (A2), we consider user signals not modeled in the signal part as additional additive Gaussian noise.

\section{SEMI-BLIND CHANNEL ESTIMATION}

We propose a blind channel estimator which requires no more than two pilot symbols per space-time slot. The proposed method is based on decorrelation of user signals. The decorrelating matched filter projects the received signal onto a subspace from which the channels of both transmitter-receiver pairs are estimated simultaneously using a low rank approximation. For a single user scenario, the decorrelating front end can be replaced with the conventional matched filter without significant performance loss.

\subsection{Blind Channel Estimation}

The decorrelating front end $\mathbf{T}^{\dagger}$ can be efficiently implemented using a state-space inversion technique which significantly reduces the complexity and storage requirement of the decorrelating receiver by exploiting the structure of the code matrix [11]. The output of the decorrelating matched filter is given by

$$
\mathbf{u}=\mathbf{T}^{\dagger} \mathbf{y}=\operatorname{diag}\left(\mathbf{I} \otimes \mathbf{H}_{1}, \cdots, \mathbf{I} \otimes \mathbf{H}_{K^{\prime}}\right) \mathbf{s}+\mathbf{n},
$$

where the $L K^{\prime} M \times 1$ vector $\mathbf{n}=\mathbf{T}^{\dagger} \mathbf{w}$ is colored. Due to the decorrelation, users are separated. Segmenting $\mathbf{u}$ to the subvectors corresponding to symbol $2 n-1,2 n$ of user $i$ gives

$$
\begin{aligned}
& \mathbf{u}_{i, 2 n-1}=\mathbf{H}_{i}\left[\begin{array}{c}
s_{i, 2 n-1}^{0} \\
s_{i, 2 n-1}^{1}
\end{array}\right]+\mathbf{n}_{2 n-1}, \\
& \mathbf{u}_{i, 2 n}=\mathbf{H}_{i}\left[\begin{array}{c}
-s_{i, 2 n-1}^{1 *} \\
s_{i, 2 n-1}^{0 *}
\end{array}\right]+\mathbf{n}_{2 n},
\end{aligned}
$$

where $n=1,2, \ldots, M / 2, \quad \mathbf{u}_{i m}$ is the $((i-1) M+m)$ th $L$ dimensional subvector of $\mathbf{u}$. Collecting data for two consecutive coded symbols of user $i$, we define a matrix $\mathbf{U}_{i n}$ as

$$
\mathbf{U}_{i n} \triangleq\left[\mathbf{u}_{i, 2 n-1} \mathbf{u}_{i, 2 n}\right]=\mathbf{H}_{i} \mathbf{S}_{i n}+\mathbf{N}_{n}
$$

where $\mathbf{N}_{n} \triangleq\left[\mathbf{n}_{2 n-1} \mathbf{n}_{2 n}\right]$, and

$$
\mathbf{S}_{i n} \triangleq\left[\begin{array}{cc}
s_{i, 2 n-1}^{0} & -s_{i, 2 n-1}^{1 *} \\
s_{i, 2 n-1}^{1} & s_{i, 2 n-1}^{0 *}
\end{array}\right]
$$


Here, $\mathbf{S}_{\text {in }}$ is a scaled unitary matrix, i.e.,

$$
\mathbf{S}_{i n} \mathbf{S}_{i n}^{H}=\mathbf{S}_{i n}^{H} \mathbf{S}_{i n}=\alpha_{n} \mathbf{I} .
$$

where $\alpha_{n}=\left|s_{i, 2 n-1}^{0}\right|^{2}+\left|s_{i, 2 n-1}^{1}\right|^{2}$. This is the key property that we exploit in developing the blind algorithm. In the noiseless case, the decomposition of $\mathbf{U}_{i n}$ gives an exact value of the channel matrix $\mathbf{H}_{i}$ up to a unknown unitary matrix factor. For noisy observation, we consider least squares estimation. The least squares estimator of the product $\mathbf{H}_{i} \mathbf{S}_{i n}$ is given by

$$
\underset{\mathbf{H}, \mathbf{S}_{i n}}{\arg \min }\left\|\mathbf{U}_{i n}-\mathbf{H}_{i} \mathbf{S}_{i n}\right\|_{F}^{2},
$$

which yields estimates of $\mathbf{H}_{i}$ and $\mathbf{S}_{\text {in }}$ (with an unknown unitary matrix factor) from the decomposition of $\mathbf{U}_{i n}$. Since we have multiple observations, using all the $\mathbf{U}_{i n}$ 's yields the channel matrix estimate

$$
\hat{\mathbf{H}}_{i}=\mathbf{V}_{i} \Sigma_{i}^{1 / 2} \mathbf{Q}_{i},
$$

where $\mathbf{Q}_{i}$ is an unknown $2 \times 2$ unitary matrix, and $\mathbf{V}_{i}$ and $\Sigma_{i}$ are given by SVD of the following covariance matrix

$$
\hat{\mathbf{R}}_{i} \triangleq \frac{2}{\alpha M} \sum_{n=1}^{M / 2} \mathbf{U}_{i n} \mathbf{U}_{i n}^{H}=\mathbf{V}_{i} \Sigma_{i} \mathbf{V}_{i}^{H},
$$

where $\alpha=\sum_{n=1}^{M / 2} \alpha_{n}$ and $\alpha_{n}$ is defined in (10). The rotational ambiguity in the above estimate can be resolved either by using pilot symbols, or by differential encoding.

We have so far assumed that the code matrix $\mathbf{T}$ of the dominant users has full column rank and therefore invertible from the left, i.e., $\mathbf{T}^{\dagger} \mathbf{T}=\mathbf{I}$. This assumption is easily satisfied if the spreading gain is large and $K^{\prime}$ is well-chosen. Under this assumption, it is clear that each user's channel is identifiable up to a rotational factor. When the spreading gain is small and we choose a large $K^{\prime}$, T can be singular. We present a general identifiability condition for the proposed method. The condition is independent of the channel parameters, and can be easily checked off-line.

Proposition 1 Let $\tilde{\mathbf{T}}_{i n} \triangleq\left[\mathbf{T}_{i, 2 n-1} \mathbf{T}_{i, 2 n}\right]$ be the matrix composed of two consecutive code matrices defined in (3) for symbol $2 n-1,2 n$ of user $i$, and $\breve{\mathbf{T}}_{i n}$ the submatrix of $\mathbf{T}$ after removing $\tilde{\mathbf{T}}_{i n}$. The channel $\mathbf{H}_{i}$ is identifiable if there exists a $n$ for user $i$ such that

$$
R\left\{\tilde{\mathbf{T}}_{i n}\right\} \bigcap R\left\{\check{\mathbf{T}}_{i n}\right\}=\{\mathbf{0}\},
$$

where $R\{\cdot\}$ denotes the range space of a matrix.

Proof: Suppose (13) holds for some $i$ and $n$. Then, the range of $\mathbf{T}$ can be represented as a direct sum of two linear subspaces, i.e., there exists a matrix $\mathbf{W}$ with $\operatorname{rank}(\mathbf{T})-\operatorname{rank}\left(\tilde{\mathbf{T}}_{i n}\right)$ linearly independent columns such that

$$
R\left\{\left[\begin{array}{ll}
\tilde{\mathbf{T}}_{i n} & \mathbf{W}
\end{array}\right]\right\}=R\{\mathbf{T}\} .
$$

Let $\mathcal{T} \triangleq\left[\begin{array}{ll}\tilde{\mathbf{T}}_{\text {in }} & \mathbf{W}\end{array}\right]$. We have, for the noiseless case,

$$
\mathcal{T}^{\dagger} \mathbf{y}=\left[\begin{array}{c}
* \\
\mathbf{h}_{i 0} s_{i, 2 n-1}^{0}-\mathbf{h}_{i 1} s_{i, 2 n-1}^{1} \\
-\mathbf{h}_{i 0} s_{i, 2 n-1}^{1 *}+\mathbf{h}_{i 1} s_{i, 2 n-1}^{0 *} \\
*
\end{array}\right],
$$

which implies that $\mathbf{H}_{i}$ is identifiable up to a rotational ambiguity.

Since (13) needs to be valid only for some $n$, the use of long codes makes the identifiability condition easier to satisfy.

\subsection{Extensions}

Since the noise $\mathbf{n}_{i m}$ after decorrelation is colored with a covariance $\mathbf{C}_{i m}$, a bias is introduced in estimation. We can apply whitening to remove the bias. The expectation of $\hat{\mathbf{R}}_{i}$ in (12) is given by

$$
\begin{aligned}
\mathbb{E}\left\{\hat{\mathbf{R}}_{i}\right\} & =\mathbf{H}_{i} \mathbf{H}_{i}^{H}+\sigma^{2} \Delta_{i} \\
\Delta_{i} & =\frac{1}{M} \sum_{m=1}^{M} \mathbf{C}_{i m}
\end{aligned}
$$

where $\mathbf{C}_{i m}$ is the $(i-1) M+m$ th diagonal block of $\mathbf{T}^{\dagger}\left(\mathbf{T}^{\dagger}\right)^{H}$ with size $L_{i} \times L_{i}$. The whitened estimator is given by

$$
\begin{aligned}
\hat{\mathbf{H}}_{i} & =\Gamma_{i} \mathbf{Q}_{i} \\
\Gamma_{i} & \triangleq \Delta_{i}^{1 / 2} \tilde{\mathbf{V}}_{i} \tilde{\Sigma}_{i}^{1 / 2}
\end{aligned}
$$

where $\Delta_{i}^{1 / 2}$ is the Cholesky factor of $\Delta_{i}\left(\Delta_{i}=\Delta_{i}^{1 / 2} \Delta_{i}^{H / 2}\right)$ and $\tilde{\mathbf{V}}_{i}, \tilde{\Sigma}_{i}$ are given by SVD of the whitened covariance matrix as o

$$
\Delta_{i}^{-1 / 2} \hat{\mathbf{R}}_{i} \Delta_{i}^{-H / 2}=\tilde{\mathbf{V}}_{i} \tilde{\Sigma}_{i} \tilde{\mathbf{V}}_{i}^{H} .
$$

For the downlink case, the data of all the users experience the same channel. We can improve the estimator performance by exploiting this. A simple approach is to combine the outer products $\hat{\mathbf{R}}_{i}$.

$$
\begin{aligned}
\hat{\mathbf{R}} & =\frac{1}{K^{\prime}} \sum_{i=1}^{K^{\prime}} \hat{\mathbf{R}}_{i}=\frac{2}{\alpha K^{\prime} M} \sum_{i=1}^{K^{\prime}} \sum_{n=1}^{M / 2} \mathbf{U}_{i n} \mathbf{U}_{i n}^{H} \\
\Delta & =\frac{1}{K^{\prime}} \sum_{i=1}^{K^{\prime}} \Delta_{i}
\end{aligned}
$$

This process further improves the performance by averaging out the noise.

For multiple receive antennas, joint estimation doesn't improve the performance when the fading channel between the transmit antennas and each receive antenna are uncorrelated, which is the design goal of antenna diversity. In this case, we can perform the above channel estimation separately for each receive antenna.

\subsection{Resolving the Rotational Ambiguity with Pilot Symbols}

The unknown unitary matrix $\mathbf{Q}_{i}$ in (11) and (15) can be solved using least squares. The least squares formulation is given by

$$
\begin{aligned}
\hat{\mathbf{Q}}_{i} & =\underset{\mathbf{Q}_{i} \in \mathbb{C}^{2 \times 2}}{\arg \min }\left\|\mathbf{U}_{i p}-\Gamma_{i} \mathbf{Q}_{i} \mathbf{S}_{p}\right\|_{F}^{2}, \\
& =\underset{\mathbf{Q}_{i} \in \mathbb{C}^{2 \times 2}}{\arg \min }\left\|\mathbf{U}_{i p} \mathbf{S}_{p}^{H}-\alpha_{p} \Gamma_{i} \mathbf{Q}_{i}\right\|_{F}^{2},
\end{aligned}
$$

under the constraint

$$
\mathbf{Q}_{i} \mathbf{Q}_{i}^{H}=\mathbf{I},
$$

where, for the example of two pilot symbols at slot front, $\alpha_{p}=$ $\left(\left|s_{i 1}^{0}\right|^{2}+\left|s_{i 1}^{1}\right|^{2}\right)$ and the pilot related matrices $\mathbf{U}_{i p}$ and $\mathbf{S}_{p}$ are given as

$$
\mathbf{U}_{i p}=\left[\mathbf{u}_{i 1}, \mathbf{u}_{i 2}\right], \quad \mathbf{S}_{p}=\left[\begin{array}{cc}
s_{i 1}^{0} & -s_{i 1}^{1 *} \\
s_{i 1}^{1} & s_{i 1}^{0 *}
\end{array}\right]
$$


The solution of the optimization problem (19) can be obtained using singular value decomposition and is given by [10]

$$
\hat{\mathbf{Q}}_{i}=\mathbf{U}_{Q} \mathbf{V}_{Q}^{H},
$$

where

$$
\alpha_{p} \Gamma_{i}^{H} \mathbf{U}_{i p} \mathbf{S}_{p}^{H}=\mathbf{U}_{Q} \Sigma_{Q} \mathbf{V}_{Q}
$$

\subsection{Detection}

Instead of resolving the unknown rotational ambiguity as in the previous section, we can employ differential decoding without the knowledge of $\mathbf{Q}_{i}$. Several differential detection schemes have been proposed [8] [9]. In [9], the differential detection relies on the assumption that the channels are zero mean and independent random variables. The detector maximizes the likelihood averaged over the channel. In this section, we give an extension of the differential detection scheme in [9] for CDMA systems with multipath channel with a deterministic assumption on the channel parameters and the knowledge of the channel subspace identified by the proposed blind method.

Consider the noiseless case. From (8), we have

$$
\begin{aligned}
\mathbf{U}_{i n} & =\mathbf{H}_{i} \mathbf{S}_{i n}=\Gamma_{i} \mathbf{Q}_{i} \mathbf{S}_{i n} \\
\mathbf{U}_{i, n+1} & =\mathbf{H}_{i} \mathbf{S}_{i, n+1}=\Gamma_{i} \mathbf{Q}_{i} \mathbf{S}_{i, n+1}
\end{aligned}
$$

where $\Gamma_{i}$ is blindly estimated via (16) (using (14), (12), and (17)). Multiplying the inverse of $\Gamma_{i}$ from the left in (23) gives

$$
\begin{aligned}
\mathbf{X}_{i n} & \triangleq \Gamma_{i}^{\dagger} \mathbf{U}_{i n}=\mathbf{Q}_{i} \mathbf{S}_{i n} \\
\mathbf{X}_{i, n+1} & =\Gamma_{i}^{\dagger} \mathbf{U}_{i, n+1}=\mathbf{Q}_{i} \mathbf{S}_{i, n+1}
\end{aligned}
$$

Since the rotational ambiguity $\mathbf{Q}_{i}$ is a unitary matrix, $\mathbf{X}$ becomes unitary if $\mathbf{S}_{i n}$ is chosen from a unitary group code, which makes the blind estimation still possible since the proposed algorithm depends only on the unitary property of the code block $\mathbf{S}_{i n}$. Applying the differential encoding scheme in [9] to $\mathbf{S}_{i n}$, differential detection is possible without knowing $\mathbf{Q}_{i}$.

Although differential decoding (DD) doesn't require channel estimation, DD usually sets the initial block to a fixed state for the ensuing differential encoding. For the proposed blind estimation method, this information is enough to estimate the entire channel, so that coherent detection can be performed. With the estimated channel, we can perform a blockwise maximum likelihood(ML) detection to obtain the symbol sequence. Rewriting eq.(7), we have

$$
\left[\begin{array}{c}
\mathbf{u}_{i, 2 n-1} \\
\mathbf{u}_{i, 2 n}^{*}
\end{array}\right]=\left[\begin{array}{cc}
\mathbf{h}_{i 0} & \mathbf{h}_{i 1} \\
\mathbf{h}_{i 1}^{*} & -\mathbf{h}_{i 0}^{*}
\end{array}\right]\left[\begin{array}{c}
s_{i, 2 n-1}^{0} \\
s_{i, 2 n-1}^{1}
\end{array}\right]+\left[\begin{array}{c}
\mathbf{n}_{2 n-1} \\
\mathbf{n}_{2 n}^{*}
\end{array}\right]
$$

ML estimates for symbol $s_{i, 2 n-1}^{0}$ and $s_{i, 2 n-1}^{1}$ are given by

$$
\left[\begin{array}{c}
\hat{s}_{i, 2 n-1}^{0} \\
\hat{s}_{i, 2 n-1}^{1}
\end{array}\right]=\mathcal{Q}\left(\left[\begin{array}{cc}
\hat{\mathbf{h}}_{i 0}^{H} & \hat{\mathbf{h}}_{i 1}^{T} \\
\hat{\mathbf{h}}_{i 1}^{H} & -\hat{\mathbf{h}}_{i 0}^{T}
\end{array}\right]\left[\begin{array}{c}
\mathbf{u}_{i, 2 n-1} \\
\mathbf{u}_{i, 2 n}^{*}
\end{array}\right]\right),
$$

where superscripts $T$ and $H$ denote transpose and Hermitian (conjugate transpose), respectively and $\mathcal{Q}$ is the quantization function which selects the symbol vector with minimum distance. The detector in (26) can be considered as a vector form of that in [5].

\section{SIMULATION RESULTS}

In this section, we present some numerical results. For channel estimation, MSE of the proposed estimator was evaluated and compared with the Cramér-Rao bound(CRB). For symbol detection, BER was used as the performance measure, and Monte Carlo runs were used to estimate the BER.

We considered a CDMA system with two transmit antennas and a single receive antenna. Five $(K=5)$ synchronous BPSK users with equal power were considered. The spreading codes for the users were randomly generated with spreading gain $G=32$ and fixed throughout the Monte Carlo simulation for MSE and BER. Since our channel model was deterministic, the channel parameters were also fixed during the Monte Carlo runs. For the CRB calculation, the symbol sequence was fixed and for MSE and BER, symbol sequences were generated randomly for each Monte Carlo run.

The channel for each TX-RX pair had three fingers $L=3$. The slot size was $M=50$ and two pilot symbols were included at the beginning of the slot of each user. These pilot symbols were used to remove the rotational ambiguity of the blind estimator. The signal-to-noise ratio (SNR) is defined by $\left(\left\|\mathbf{h}_{0}\right\|^{2}+\right.$ $\left.\left\|\mathbf{h}_{1}\right\|^{2}\right) G E_{c} / \sigma^{2}$ where $E_{c}$ is the chip energy and $\sigma^{2}$ is the chip noise variance.

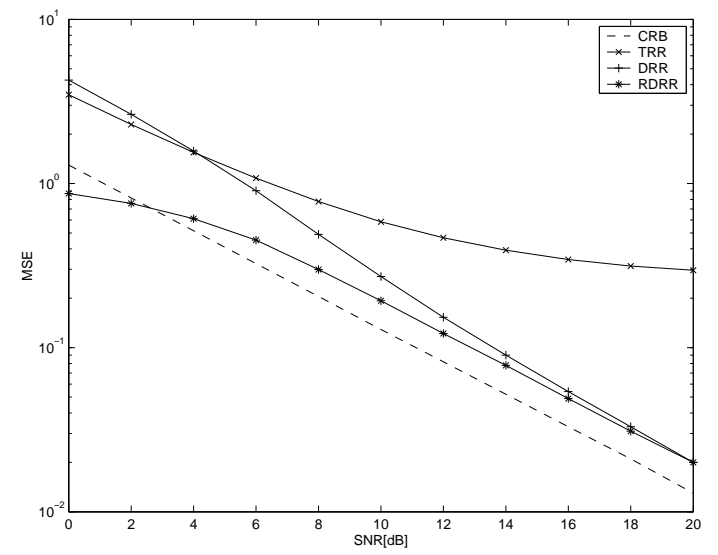

Fig. 2. Channel MSE vs. SNR. Two pilot symbols per user.

We compared the mean square error performance of the proposed channel estimator using the decorrelating front end (DRR) and regularized decorrelating front end (RDRR) with the CRB and the conventional training based algorithm (TRR) based on the standard matched filter. The regularized decorrelating front end which is given as $\left(\mathbf{T}^{H} \mathbf{T}+\sigma^{2} \mathbf{I}\right)^{-1} \mathbf{T}^{H}$ was used to mitigate the noise enhancement of the normal inversion. It requires the estimation of noise power. In this simulation, the true noise power was used. Due to the two pilot symbols inserted for rotational ambiguity resolution, we used the semi-blind $\mathrm{CRB}$ with a deterministic assumption on data symbols [17]. For the training based method, a least-squares channel estimate was obtained using observations corresponding to the pilot symbols. Fig. 2 shows the MSE performance. We observe that as SNR increases, the MSE of the conventional method (TRR) deviates from the CRB due to the multiaccess interference. The DRR, on the other hand, tracks the CRB closely 
at high SNR. The high MSE of DRR at low SNR is due to the enhancement of the noise by the decorrelating (zero-forcing) receiver. The better performance of RDRR over the unbiased CRB is due to the noise reduction in regularization which makes the estimator biased.

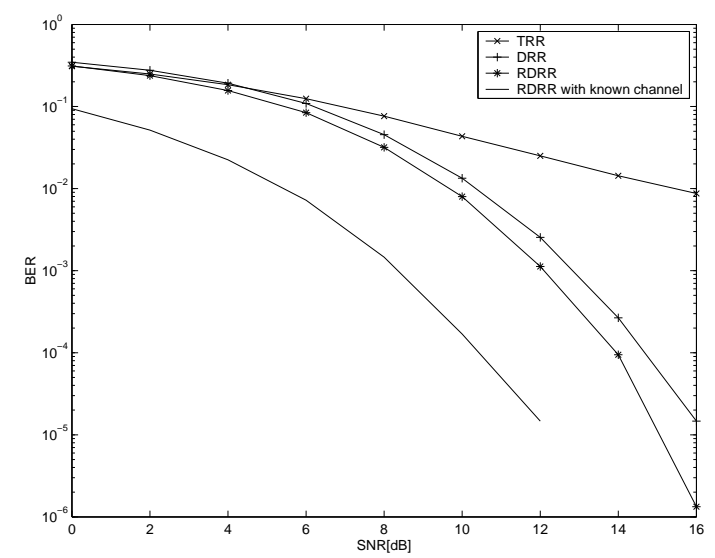

Fig. 3. BER vs. SNR. Two pilot symbols per slot.

We evaluated the BER performance of the ML detector in Section 3 with decorrelating, regularized decorrelating, and Hermitian $\mathbf{T}^{H}$ front ends, using RDRR with true channel as the performance bound. Fig. 3 shows the BER performance averaged over users. We observe a $4 \mathrm{~dB}$ loss at BER of $10^{-3}$ due to channel estimation errors. The proposed method shows a significant improvement over the conventional RAKE receiver as SNR increases: the BER improvement with respect to TRR is several orders of magnitude at $14 \mathrm{~dB}$ SNR. The well known floor effect of the conventional receiver is due to multiaccess interference.

\section{CONCLUSION}

We propose a new semi-blind channel estimation technique for space-time coded wideband CDMA systems which requires only two pilot symbols per slot. A new identifiability condition is established. The proposed method identifies the channel of each transmit-receive pair simultaneously exploiting the subspace structure of WCDMA signals and the orthogonality of space-time codes with a few pilot symbols. The decoding procedure for differential modulation for CDMA systems is given.

\section{REFERENCES}

[1] G. J. Foschini and M. J. Gans,'On the limits of wireless communications in a fading environment when using multiple antennas," Wireless Personal Communications, vol. 6, no. 3, pp. 311-335, 1998.

[2] G. J. Foschini, "Layered space-time architecture for wireless communication in a fading environment when using multiple antennas," Bell Labs Technical Journal, vol. 1, no. 2, pp. 4159, Autumn, 1996.

[3] P. W. Wolniansky, G. J. Foschini, G. D. Golden, and R. A. Valenzuela," V-BLAST: an architecture for realiz- ing very high data rates over the rich-scattering wireless channel," International Symposium on Signals, Systems, and Electronics, pp. 295-300, 1998.

[4] H. Huang, H. Viswanathan, and G. J. Foschini, “ Achieving high data rates in CDMA systems using BLAST techniques," Proc. Globecom, vol.5, pp. 2316-2320, 1999.

[5] S. M. Alamouti, "A simple transmit diversity technique for wireless communications," IEEE Journal on Selected Areas in Communications, vol.16, no.8, pp.1451-1458, Oct. 1998.

[6] V. Tarokh, H. Jafarkhani, and A. R. Calderbank, "Space-time block codes from orthogonal designs," IEEE Trans. Information Theory, vol. 45, pp.1456-1467, July, 1999.

[7] B. M. Hochwald and T. L. Marzetta, "Unitary spacetime modulation for multiple antenna communications in Rayleigh flat fading," IEEE Trans. Information Theory, vol. 46, no. 2, pp. 543-564, March 2000.

[8] V. Tarokh and H. Jafarkhani, "A differential detection scheme for transmit diversity," IEEE Journal on Selected Areas in Communications, vol. 18, no. 7, pp. 1169-1174, July 2000.

[9] B. L. Hughes, "Differential space-time modulation," IEEE Wireless Communications and Networking Conference, vol. 1, pp.145 -149, 1999.

[10] G. Golub and C. V. Loan, Matrix Computations. Baltimore, Maryland: The Johns Hopkins University Press, 1990.

[11] L. Tong, A. van der Veen, P. Dewilde, and Y. Sung, "Blind decorrelating rake receiver for long code WCDMA," Tech. Rep. ACSP-02-01, Cornell University, Feb. 2002.

[12] L. Tong, A. van der Veen, and P. Dewilde, "Channel estimation for long-code WCDMA," Proc. ISCAS 2002, 651-654, vol III, 2002.

[13] Y. Sung and L. Tong, "Channel tracking for fast fading long code WCDMA,” Proc. MILCOM 2002, Oct. 2002.

[14] Z. Xu and M.K. Tsatsanis, "Blind channel estimation for multiuser CDMA systems with long spreading codes," Proc. ICASSP, 2531-34, 1999.

[15] X. Mestre and J. Fonollosa, "ML approaches to channel estimation for pilot-aided multirate DS/CDMA systems," IEEE Trans. Sig. Proc., 50(3), 696-709, March 2002. See also Proc. ICASSP, 2201-04, Salt Lake City, USA, 2001.

[16] J. Luo and J.R. Zeidler, "Error probability performance for WCDMA systems with multiple transmit and receive antennas in correlated Nakagami fading channels," Proc. Globecom, 1220-23, San Antonio, USA, 2001.

[17] E. De Carvalho and D. T. M. Slock, "Cramer-Rao bounds for semi-blind, blind and training sequence based channel estimation," Proc. IEEE SP Workshop on SPAWC, pp. 129132, 1997. 